\title{
Stem cells in the limelight
}

\section{Stem cell biology has emerged as one of the most exciting areas of basic and biomedical research. This month, we launch a series of specially commissioned articles that discuss recent advances and challenges in this field.}

Stem cells are unique in their capacity to self-renew and differentiate into a wide array of more specialized cells. Whereas embryonic stem cells - cells isolated from the inner cell mass of blastocysts - can give rise to all three germ layers, tissue-resident adult stem cells serve primarily to replenish their host tissues and organs during homeostasis, injury and disease. Given their important roles in the organism, stem cell dysfunction has been linked to ageing and diseases such as cancer. These properties render stem cells, and their more differentiated derivatives, attractive models for studying development and disease, and a potential source for cell-replacement therapies. Contributing towards a comprehensive understanding of stem cell biology and its relevance to normal physiology, ageing and disease is central to the purview of Nature Cell Biology.

With this issue, we are pleased to introduce our Series on Stem Cell Biology, which launches with two Review articles. In the first, Maike Sander and colleagues discuss the evidence for and against the roles of stem cells and differentiated cells in maintenance and repair of the liver and pancreas. This is followed by a Review by Nick Barker and colleagues on the organoid systems that have been established using stem cells from different tissues, their value for studying development and disease, and their potential future uses in personalized medicine. These two articles are accompanied by a Commentary by Michael Yaffe and colleagues on the important issue of authentication of induced pluripotent stem cells. Next month's issue will include a Perspective by Jane Visvader and Hans Clevers discussing how stem cell hierarchies arise in different tissues, and how their plasticity is regulated after injury. Over the subsequent months, we hope to present articles on the influences of different metabolic states on stem cell functions during homeostasis, regeneration and physiological ageing, and on the challenges and recent progress made towards the de novo generation of haematopoietic stem cells from human pluripotent stem cells.

The Series is hosted on a dedicated page of our website. With this selection of commissioned articles, we do not intend to provide a comprehensive overview of this thriving field of research, but rather to inform our readers about emerging themes, recent developments and ongoing challenges. The Series is accompanied by an online library of selected related research articles published over the past 18 months by Nature Cell Biology as well as other Nature journals. This collection highlights how the wide scope of this journal covers broad themes in the stem cell field, aiming to provide insights into fundamental questions of their underlying biology and their roles in development and disease.

Central to using stem cells and their derivatives to study development, regeneration and disease is the understanding of their intrinsic properties - pluripotency and self-renewal - and the mechanisms underlying their regulation. This year marks the tenth anniversary of a major breakthrough in this field: the first report of somatic cell reprogramming, the process by which differentiated cells can be reverted into a pluripotent state. This is a thriving area of research, as it holds huge promise for the modelling of human disease. However, a full understanding of how pluripotency and somatic cell reprogramming are controlled is still lacking, and so the cell biological, molecular and epigenetic mechanisms underlying these properties are key areas of interest here at Nature Cell Biology.

A rapidly evolving field within the scope of this journal is the identification and characterization of tissue-resident stem cells. Recent technological advances have permitted the single-cell analysis of stem and progenitor cells (in culture or isolated from tissues) to explore the heterogeneity, hierarchies and dynamics of cell populations. Complementary approaches, such as the in vivo analysis of clonal tissue stem cell dynamics using lineage tracing, can provide valuable insights into the roles of these cells and their derivatives in homeostasis, disease and regeneration. The mechanisms that regulate the plasticity of stem cells and their more differentiated progeny in development, after injury and in disease states such as cancer is also a fascinating topic covered by Nature Cell Biology.

Another area of active research related to these subjects that this journal aims to cover is how the environment shapes tissue stem cell properties. Revealing the strategies by which the niche in which stem cells reside controls their maintenance and behaviour in different situations, and how metabolic states, the immune system and ageing affect stem cell attributes, is crucial for elucidating the function of these cells in the overall physiology of the organism.

In recent years, stem cells have been used to generate in vitro organoids representative of different tissues. Such approaches and their relevance to modelling normal tissue architecture and disease states, including cancer, are topics of interest to this journal. However, although we acknowledge the importance of demonstrating the use of stem cells and their derivatives in treating diseases and injury, or for engineering tissues, we consider studies that focus more on aspects of translational and biotechnological research as more peripheral to the journal's purview.

Although the themes highlighted here are by no means a detailed discussion of the scope of our journal in stem cell biology, they are intended to underscore our strong commitment to continue being a forum for the publication of important advances in this field. We hope that you will find the commissioned articles and archive of research papers in our Series on Stem Cell Biology exciting and informative, and we thank our authors and referees for their contributions. 